\title{
A Analysis on Queuing System with Setup Time in Revamp Process
}

\author{
P. Karunakaran, S. Maragathasundari
}

\begin{abstract}
In this paper, we study about a M/G/1 Queuing model with single stage of service. Service interrupts during the time of service. The server does not get into the repair process immediately. It gets into a Set up time stage for the prior work to be done. On completion of set up stage service, the server will get into the repair process consisting of two stages, in which first stage is compulsory and the second stage of service is optional. For the model defined, we get the steady state results in closed form in terms of the probability generating functions and all the other execution performance measures of the model defined.
\end{abstract}

Keywords: Setup time, compulsory service, Optional service, Revamp process

\section{INTRODUCTION}

Q ueuing theory was developed to provide models to predict the behavior of systems that attempt to provide service for randomly arising demands. Queuing system of bulk arrival model with optional service examined by [1]. "Reference [2] investigated Poisson input queuing system with startup time".

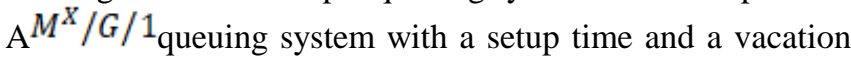
period studied by [3]. "Reference [6] inspected classification of queuing models for a workstation with interruptions". Queuing model of optional type of service with service stoppage and revamp process in web hosting queuing well analyzed by [7]. "Reference [10] studied revamping quality of service of video streaming over wireless LAN". "Reference [4] studied a batch arrival Poisson queue with a random setup time". "Reference [5] examined a batch service queuing system with multiple vacations". "Reference [9] investigated on the optimal control two queues with server setup times and its analysis". "Reference [8] analyzed queue in Multichannel V2I Communications".

\section{MATHEMATICAL DESCRIPTION OF THE QUEUEING MODEL}

Customers arrival follows Poisson procedure, Let $\lambda_{g} d t$ be the first order probability that a customer's arrives at the system during a short duration of time $(t, t+d t)$, where

Revised Manuscript Received on December 15, 2019.

* Correspondence Author

P. Karunakaran, Department of Mathematics, Kalasalingam Academy of Research and Education, Krishnankovil, Tamilnadu. Email: karunamath1986@gmail.com

S. MaragathaSundari*, Department of Mathematics, Kalasalingam Academy of Research and Education, Krishnankovil, Tamilnadu. Email: maragatham01@gmail.com

$\lambda_{g}>0$ the mean landing rate of the customer is.

The administration time pursues general distribution.

Service follows distribution function as $J_{1}^{*}(x)$ and density function $l_{1}^{*}(x)$. Let $\gamma_{g}(x) d x$ be the conditional density function. Hence we have

$$
\gamma_{g}(x)=\frac{L_{1}^{*}(x)}{1-J_{1}^{*}(x)}, l_{1}^{*}(x)=\gamma_{g}(x) e^{-\int_{0}^{s} V_{g}(s) d s}
$$

For set up time,

$\gamma_{m}(x)=\frac{l_{2}^{*}(x)}{1-J_{2}^{*}(x)} \quad, l_{2}^{*}(x)=\gamma_{m}(x) e^{-\int_{0}^{s} \gamma_{m}(s) d s}$

For compulsory stage 1 service,

$\gamma_{n_{a}}(x)=\frac{l_{3}^{*}(x)}{1-f_{3}^{*}(x)} \quad, l_{3}^{*}(x)=\gamma_{n_{a}}(x) e^{-\int_{0}^{s} \gamma_{n_{a}}(s) d s}$

For optional service stage 2,

$\gamma_{n_{b}}(x)=\frac{l_{4}^{*}(x)}{1-J_{4}^{*}(x)} \quad, l_{4}^{*}(x)=\gamma_{n_{b}}(x) e^{-\int_{0}^{s} \gamma_{n_{b}}(s) d s}$

\section{GOVERNING EQUATIONS OF THE MODEL}

Steady State Conditions Overseeing the Framework

$$
\begin{aligned}
& \frac{\partial}{\partial x} G_{n}(x)+\left(\lambda_{g}+\gamma_{g}(\mathrm{x})+\psi\right) G_{n}(x)=\lambda_{g} G_{n-1}(x) \\
& \frac{\partial}{\partial x} G_{0}(x)+\left(\lambda_{g}+\gamma_{g}(\mathrm{x})+\psi\right) G_{0}(x)=0 \\
& \frac{\partial}{\partial x} M_{n}(x)+\left(\lambda_{g}+\gamma_{m}(\mathrm{x})\right) M_{n}(x)=\lambda_{g} M_{n-1}(x) \\
& \frac{\partial}{\partial x} M_{0}(x)+\left(\lambda_{g}+\gamma_{m}(\mathrm{x})\right) M_{0}(x)=0 \\
& \frac{\partial}{\partial x} R_{n}^{(a)}(x)+\left(\lambda_{g}+\gamma_{n_{a}}(\mathrm{x})\right) R_{n}^{(a)}(x)=\lambda_{g} R_{n-1}^{(a)}(x) \\
& \frac{\partial}{\partial x} R_{0}^{(a)}(x)+\left(\lambda_{g}+\gamma_{n_{a}}(\mathrm{x})\right) R_{0}^{(a)}(x)=0 \\
& \frac{\partial}{\partial x} R_{n}^{(b)}(x)+\left(\lambda_{g}+\gamma_{n_{b}}(\mathrm{x})\right) R_{n}^{(b)}(x)=\lambda_{g} R_{n-1}^{(b)}(x) \\
& \frac{\partial}{\partial x} R_{n}^{(b)}(x)+\left(\lambda_{g}+\gamma_{n_{b}}(\mathrm{x})\right) R_{n}^{(b)}(x)=0 \\
& \lambda^{g} E=\int_{0}^{\infty} G_{0}(x) \gamma_{g}(\mathrm{x}) \mathrm{dx}+(1-l) \int_{0}^{\infty} R_{n}^{(a)}(x) \gamma_{n_{a}}(\mathrm{x}) \mathrm{dx}+ \\
& \int_{0}^{\infty} R_{n}^{(b)}(x) \gamma_{n_{b}}(\mathrm{x}) \mathrm{dx}
\end{aligned}
$$

\section{BOUNDARY CONDITIONS}

The above set of equations is to be solved under the following boundary conditions at $x=0$ and for $x \geq 1$ $G_{n}(0)=$

$$
\begin{aligned}
& (1-l) \int_{0}^{\infty} R_{n+1}^{(a)}(x) \gamma_{n_{a}}(\mathrm{x}) \mathrm{dx}+\int_{0}^{\infty} R_{n+1}^{(b)}(x) \gamma_{n_{b}}(\mathrm{x}) \mathrm{dx}+ \\
& \lambda_{g} E \int_{0}^{\infty} G_{n+1}(x) \gamma_{g}(\mathrm{x}) \\
& M_{n}(0)=\psi \int_{0}^{\infty} G_{n}(x) d x \text {. } \\
& R_{n}^{(a)}(0)=\int_{0}^{\infty} M_{n}(x) \gamma_{m}(\mathrm{x}) \mathrm{dx} \text {. }
\end{aligned}
$$

$$
R_{n}^{(b)}(0)=l \int_{0}^{\infty} R_{n}^{(a)}(x) \gamma_{n_{a}}(\mathrm{x}) \mathrm{dx}
$$

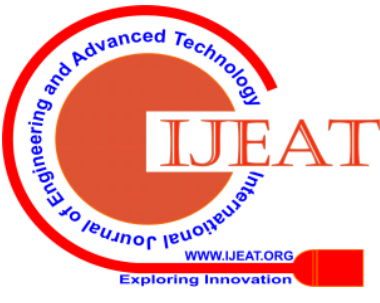


multiply (1) by $z^{n}$ and sum over $n$ from 1 to, adding to (2), we get

$\frac{\partial}{\partial x} G_{n}(x, z)+\left(\lambda_{g}-\lambda_{g} z+\gamma_{g}(\mathrm{x})+\Psi\right) G_{n}(x, z)=0$.

$\int_{0}^{x}(14)$ Gives,

$G_{n}(x, z)=G_{n}(0, z) e^{-\left(\lambda_{g}-\lambda_{g} z+\psi\right)-\int_{0}^{x} y_{g}(\mathrm{t}) \mathrm{dt}}$.

$\int_{0}^{x}(15) d x$ By parts $\Rightarrow$

$G_{n}(z)=G_{n}(0, z)\left[\frac{1-J_{1}^{*}\left(\lambda_{g}-\lambda_{g} z+\psi\right)}{\left(\lambda_{g}-\lambda_{g} z+\psi\right)}\right]$.

Multiply both sides of (15) by $\gamma_{g}(\mathrm{x}) \mathrm{dx}$ and integrating by parts, we get

$\int_{0}^{\infty} G_{n}(z) \gamma_{g}(\mathrm{x}) \mathrm{dx}=G_{n}(0, z) J_{1}^{*}\left(\lambda_{g}-\lambda_{g} z+\psi\right)$.

Applying the same process of study for the remaining equations (3) - (8), we get

$M_{n}(z)=M_{n}(0, z)\left[\frac{1-J_{2}^{z}\left(\lambda_{g}-\lambda_{g} z\right)}{\left(\lambda_{g}-\lambda_{g} z\right)}\right]$.

$\int_{0}^{\infty} M_{n}(x, z) \gamma_{m}(\mathrm{x}) \mathrm{dx}=M_{n}(0, z) J_{2}^{*}\left(\lambda_{g}-\lambda_{g} z\right)$.

$R_{n}^{(a)}(z)=R_{n}^{(a)}(0, z)\left[\frac{1-J_{3}^{*}\left(\lambda_{g}-\lambda_{g} z\right)}{\left(\lambda_{g}-\lambda_{g} z\right)}\right]$.

$\int_{0}^{\infty} R_{n}^{(a)}(x, z) \gamma_{n_{a}}(\mathrm{x}) \mathrm{dx}=R_{n}^{(a)}(0, z) J_{3}^{*}\left(\lambda^{g}-\lambda^{g} z\right)$.

$R_{n}^{(b)}(z)=R_{n}^{(b)}(0, z)\left[\frac{1-J_{4}^{*}\left(\lambda_{g}-\lambda_{g} z\right)}{\left(\lambda_{g}-\lambda_{g} z\right)}\right]$.

$\int_{0}^{\infty} R_{n}^{(b)}(x, z) \gamma_{n_{b}}(\mathrm{x}) \mathrm{dx}=R_{n}^{(b)}(0, z) J_{4}^{*}\left(\lambda_{g}-\lambda_{g} z\right)$.

Using Supplementary variable method and using (17), (21),

(23) in (10), we get

$G_{n}(0, z)=\frac{\lambda_{g} E z-\lambda_{g} E}{z-J_{1}^{*}(R)+\left[\frac{1-J_{1}^{*}(R)}{R}\right] J_{2}^{*}(C) J_{3}^{*}(C)\left[\psi(1-l)+l J_{4}^{*}(C)\right]}$.

Where $\quad R=\lambda_{g}-\lambda_{g} z+\Psi, \quad C=\lambda_{g}-\lambda_{g} z$.

\section{LIKELIHOOD CREATING CAPACITY OF THE LINE ESTIMATE}

Let $\overline{A_{k}}(z)$ be the p.g.f of the line length such that

$$
\begin{aligned}
& \overline{A_{k}}(z)=G_{n}(z)+M_{n}(z)+R_{n}^{(a)}(z)+R_{n}^{(b)}(z) . \\
& \overline{A_{k}}(z)=\frac{\left[\frac{1-J_{1}^{*}(R)}{R}\right] E\left\{\begin{array}{c}
\left(\lambda_{g} z-\lambda_{g}\right)\left(1+J_{4}^{*}(C) l J_{3}^{*}(C) J_{2}^{*}(C) \psi\right) \\
\left.-\left(\psi\left(1-J_{2}^{*}(C)\right)+\psi\left(1-J_{3}^{*}(C) J_{2}^{*}(C)\right)\right)\right\}
\end{array}\right.}{z-J_{1}^{*}(R)+\left[\frac{1-J_{1}^{*}(R)}{R}\right] J_{3}^{*}(C) J_{2}^{*}(C)\left[\psi(1-l)+l J_{4}^{*}(C)\right]} .
\end{aligned}
$$

\section{NORMALIZATION CONDITION}

$F(1)+E=1$ Gives the idle time $S$ and the Utilization factor. Idle time $S=\frac{D^{\prime}(1)}{D^{\prime}(1)+N^{\prime}(1)}$

Utilization factor, $\rho=1-E$.

$F(1)=\lim _{z \rightarrow 1} F(z)=\frac{0}{0}$,indeterminant form.

Hence applying L'Hopital's rule, we get

$F(1)=\frac{D^{I}(1)}{D^{I}(1)+N^{I}(1)}$.

\section{SYSTEM QUEUE PERFORMANCE MEASURES}

Let $L_{q}$ a chance to demonstrate the reliable state typical number of customers in the line. By then

$L_{q}=\left.\frac{d}{d z} \overline{A_{k}}(z)\right|_{z=1}=\left.\frac{d}{d z}\left\{\frac{N(Z)}{D(Z)}\right\}\right|_{z=1}$

Where $N(Z)$ and $D(Z)$ are the numerator and denominator of (25).

Since $\overline{A_{k}}(z)=\frac{0}{0}$ at $z^{=1}$, we utilize two fold separation and get

$$
\begin{aligned}
& L_{q}=\lim _{z \rightarrow 1} \frac{d}{d z} \overline{A_{k}}(z)=\frac{D^{\prime}(1) N^{\prime \prime}(1)-D^{\prime \prime}(1) N^{\prime}(1)}{2\left(D^{\prime}(1)\right)^{2}} . \\
& N^{\prime}(1)=\left[1-J_{1}^{*}(\psi)\right]\left\{\lambda_{g}\left(E\left(J_{2}\right)+E\left(J_{3}\right)\right)\right\} \text {. } \\
& N^{\prime \prime}(1)=-J_{1}^{\varepsilon^{\prime}}(\psi)\left(E\left(J_{1}\right)+E\left(J_{3}\right)\right) \lambda_{g}(1+\psi)+ \\
& \left(1-J_{1}^{*}(\psi)\right)\left\{-2\left(\lambda_{g}\right)^{2} E\left(J_{4}\right) l+\left[E\left(J_{2}^{2}\right)\left(\lambda_{g}\right)^{2}+\right.\right. \\
& \left.\left.\left(\lambda_{g}\right)^{2} E\left(J_{3}^{2}\right)+2 E\left(J_{2}\right) E\left(J_{3}\right)\left(\lambda_{g}\right)^{2}\right]\right\} \\
& D^{\prime}(1)=1-\lambda_{g} J_{1}^{*^{\prime}}(\psi)-J_{1}^{\varepsilon^{\prime}}(\psi)[\psi(1-l)+l]+ \\
& {\left[\frac{1-J_{1}^{*}(\psi)}{\psi}\right][\psi(1-l)+l] \lambda_{g}\left(E\left(J_{2}\right)+E\left(J_{3}\right)\right)+} \\
& {\left[\frac{1-J_{1}^{*}(\psi)}{\psi}\right] \lambda_{g} l E\left(J_{4}\right)} \\
& D^{\prime \prime}(1)=-J_{1}^{s^{\prime \prime}}(\psi)\left(\lambda_{g}\right)^{2}+J_{1}^{\prime \prime \prime}(\psi) \lambda_{g}[\psi(1-l)+l]- \\
& I_{1}^{*^{\prime}}(\psi) \lambda_{g} l E\left(J_{4}\right)+\left[\frac{1-J_{1}^{*}(\psi)}{\psi}\right]\left[-\lambda_{g} l E\left(J_{4}\right)\right] \lambda_{g}\left(E\left(J_{2}\right)+\right. \\
& \left.E\left(J_{3}\right)\right)+\left[\frac{1-J_{1}^{*}(\psi)}{\psi}\right][\psi(1-l)+l]\left[E\left(J_{3}^{2}\right)+E\left(J_{2}^{2}\right)+\right. \\
& \left.2 E\left(J_{2}\right) E\left(J_{3}\right)\right]\left(\lambda_{g}\right)^{2}-J_{1}^{*^{\prime}}(\psi) \lambda_{g} l E\left(J_{4}\right)+ \\
& {\left[\frac{1-J_{1}^{*}(\psi)}{\psi}\right] l\left(\lambda_{g}\right)^{2}\left[E\left(J_{4}^{2}\right)+E\left(J_{4}\right)\left(E\left(J_{2}\right)+E\left(J_{3}\right)\right)\right]}
\end{aligned}
$$

Substituting (30) - (33) in (29), we obtain $L_{q}$ and all the other measures using Little's formula: $W_{q}=\frac{L_{q}}{\lambda},=\frac{L}{\lambda}$, $L=L_{q}+\rho$.

\section{NUMERICAL JUSTIFICATION OF THE MODEL}

Here we consider the service time to follow exponential distribution.

The values are collected accordingly:

$\gamma_{g}=2.5, \gamma_{m}=3, \gamma_{n_{a}}=3.5, \gamma_{n_{b}}=4, l=0.6$,

$I_{1}^{*}(\psi)=\frac{\gamma_{g}}{\gamma_{g}+\psi}, J_{1}^{z^{\prime}}(\psi)=\frac{-\gamma_{g}}{\left(\gamma_{g}+\psi\right)^{2}}, J_{1}^{z^{\prime \prime}}(\psi)=\frac{2 \gamma_{g}}{\left(\gamma_{g}+\psi\right)^{3}}$,

$E\left(J_{2}\right)=\frac{1}{\gamma_{m}}, E\left(J_{2}^{2}\right)=\frac{2}{\left(\gamma_{m}\right)^{2}}, E\left(J_{3}\right)=\frac{1}{\gamma_{n_{a}}}$,

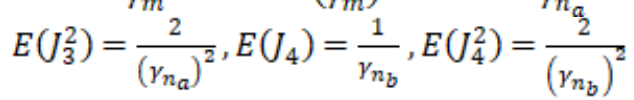

Table I

Effect of change of $(\psi=1.5,2,2.5,3,3.5)$

\begin{tabular}{|l|l|l|l|l|l|}
\hline $\mathrm{Q}$ & $\rho$ & $L_{q}$ & $L$ & $W_{q}$ & $W$ \\
\hline 0.7759 & 0.2241 & 0.1294 & 0.3535 & 0.0462 & 0.1263 \\
\hline 0.7364 & 0.2636 & 0.1441 & 0.4077 & 0.0515 & 0.1456 \\
\hline 0.7058 & 0.2942 & 0.1523 & 0.4465 & 0.0544 & 0.1595 \\
\hline 0.6815 & 0.3185 & 0.1568 & 0.4753 & 0.0560 & 0.1698 \\
\hline 0.6621 & 0.3379 & 0.1589 & 0.4968 & 0.0568 & 0.1774 \\
\hline
\end{tabular}

Published By:

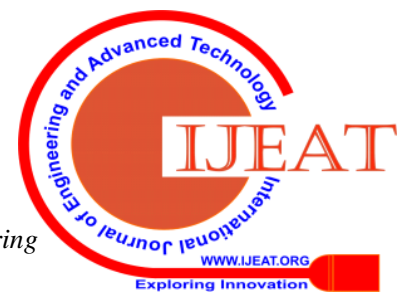




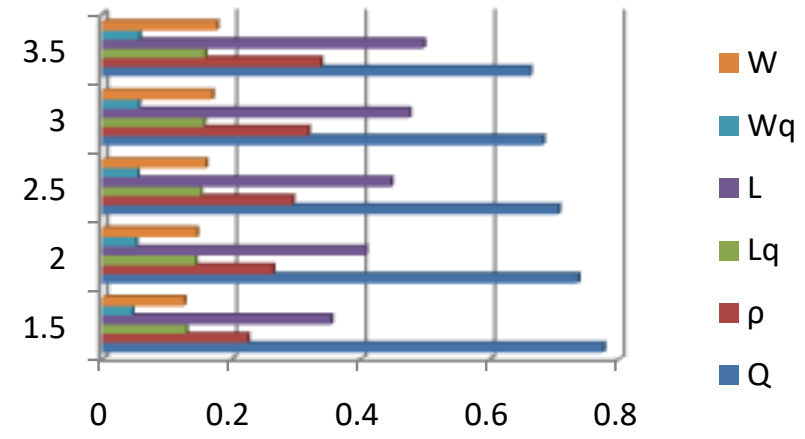

Fig. 1 Effect of change of $\psi$

From Table $I$ it is clear that if the Service interruption increases it leads to an increase in all the performance measures. Since the Service interruption gets increased the idle time gets decreased and utilization factor is increased. Waiting time of queue and waiting time of server are also increased when the service interruption gets increased. When we include maintenance work we will avoid waiting time of the queue and also waiting time of server.

Table II

Effect of change of $(l=0.2,0.4,0.6,0.8,1)$

\begin{tabular}{|l|l|l|l|l|l|}
\hline $\mathrm{Q}$ & $\rho$ & $L_{q}$ & $L$ & $W_{\text {q }}$ & $W$ \\
\hline 0.7795 & 0.2205 & 0.2511 & 0.4716 & 0.0897 & 0.1684 \\
\hline 0.7777 & 0.2223 & 0.1909 & 0.4132 & 0.0682 & 0.1476 \\
\hline 0.7759 & 0.2241 & 0.1294 & 0.3535 & 0.0462 & 0.1263 \\
\hline 0.7740 & 0.2260 & 0.0666 & 0.2926 & 0.0238 & 0.1045 \\
\hline 0.7721 & 0.2279 & 0.0025 & 0.2304 & 0.0009 & 0.0823 \\
\hline
\end{tabular}

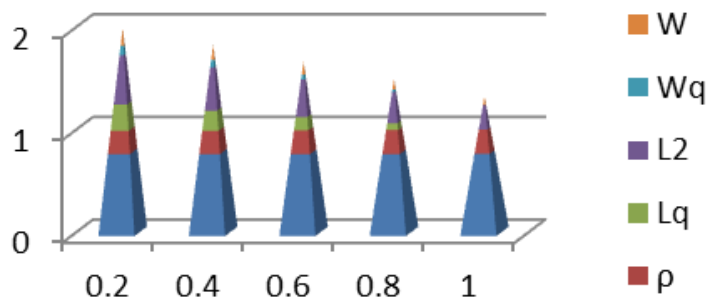

Fig. 2 Effect of change of $l$

Table 2 indicates that, as the probability of oiptonal service gets increased, the idle time is decreased but utilization factor is increased. Since the optional service increases it leads to decrease in all the performance measures.

\section{CONCLUSION}

In this paper, we have examined a single service. When the service interruption occurs the server gets into a setup time with two stages. The first stage of service is compulsory and the second stage is optional. This paper clearly analyses the steady state results and some queuing performance measures. Further this model can be extended by adding the concept of balking, reneging, feedback service, extended vacation etc.

\section{REFERENCES}

[1] K. Anitha , S. Maragathasundari and M. Bala , "Queuing system of bulk arrival model with optional services in third stage and two different vacation policies", International Journal of Mathematics and its applications, Vol. 5, No. 4, pp. 711-721, 2017.

[2] A. Borthakur, J. Medhi , R. Gohain (1987), "Poisson Input queuing system with startup time and under control operating policy ", Elsevier, Vol. 14, Ni. 1, pp. 33-40.

[3] GautamChoudhury, "A $M^{X} / G / 1$ queuing system with a setup Period and a vacation period”, Springer, Vol. 36, No. 1-3, pp. 23-38, 2000.

[4] Gautham Choudhury, "On a batch arrival Poisson queue with a random setup time and vacation period", Elsevier, Vol. 25, No. 12, pp. 1013-1026, 1998.

[5] M. Haridass and R. Arumuganathan , "A batch service queuing system with multiple vacations, setup time and server's choice of admitting reservice", International Journal Operational Research, Vol. 14, No. 2, pp. 156-186, 2012.

[6] Kan $\mathrm{Wu}$, "Classification of queuing models for a workstation with interruptions: a review”, International Journal of Production Research, Vol. 52, No. 3, pp. 902-917, 2013.

[7] S. Maragathasundari and Miriam Cathy Joy, "Queuing model of optional type of service with service stoppage and revamp process in web hosting queuing", International Journal of Knowledge Management in Tourism and Hospitality, Vol. 1, No. 2, pp. 241-262, 2017.

[8] Maurice Khabbaz, MazenHasna, Chadi M. Assi, Ali Ghrayeb , "Modelling and analysis of an Infrastructure Service request Queue in Multichannel V2I Communications", IEEE, Vol. 15, No. 3, pp. 1155-1167, 2014.

[9] MichaHofri and Keith W. Ross, "On the Optimal Control Two Queues with Server Setup Times and its Analysis", Society for Industrial and Applied Mathematics, Vol. 16, No. 2, pp.399-420, 2006.

[10] RohanThakkar and Shantanu Mane, "Revamping Quality Of Service of Video Streaming over wireless LAN", International Journal of Research in Engineering and Technology, Vol. 3, No. 7, pp.352-359, 2014.

\section{AUTHORS PROFILE}

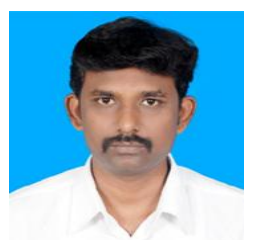

Mr. P. Karunakaran pursued his B.Sc degree from Ayya Nadar Janaki Ammal College, Sivakasi in 2009. He got his M.Sc degree from Ayya Nadar Janaki Ammal College, Sivakasi in 2012. He obtained his M.Phil. degree from Ayya Nadar Janaki Ammal College, Sivakasi in 2013. He had 6 years of teaching experience. Now he is doing research area in Queuing Theory in Kalasalingam Academy of Research and Education, Anand Nagar, Krishnankoil-626126, Tamilnadu, India.

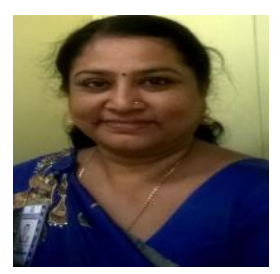

Dr. S. Maragatha Sundari acquired her B.Ed degree from V.O.C Teachers College, Tuticorin in 1993. She got her M.Sc. in Mathematics from Manonmaniam Sundaranar University; Tirunelveli in 1995 and her M.Phil. degree from Madurai Kamaraj University, Madurai in 2003. She did her Ph.D. in Sathyabama University, Chennai, India. She has more than 17 years of instructing knowledge. She has distributed more than 50 inquire about papers in national and international journals. She has displayed and distributed papers at national and international conferences. She is right now filling in as an Associate Professor in the Department of Mathematics in Kalasalingam Academy Of Research And Education, Anand Nagar, Tamilnadu, Krishnankovil-626126, India. In addition, she is doing her research guidance for five research scholars. 